\title{
Design of experiment for the validation of ATP/ATC odometry algorithms
}

\author{
M. Malvezzi ${ }^{1}$, G. Cocci $^{2} \&$ A. Tarasconi ${ }^{2}$ \\ ${ }^{I}$ Department of Energetics S. Stecco, University of Florence, Firenze, \\ Italy \\ ${ }^{2}$ Trenitalia S.p.A., Firenze, Italy
}

\begin{abstract}
The safety and efficiency of a railway control systems depend on many factors, one of these being the precision of train position and speed estimation. The knowledge of the estimation precision is important in the developing phase of the procedure and in the integration with the automatic train protection system, in order to properly assess the safety margins used to define the system intervention. The estimation is performed by the so-called odometry system, which includes a series of sensors and a software procedure. The data set used to test the algorithm has to represent all the real conditions that will be met in normal conditions. The criteria used to define the set of tests is based on the DOE (Design Of Experiment) technique, which allows one to obtain a compromise between the number of needed tests and the complete reproduction of possible cases. Once the set of tests are defined, they can be reproduced on a test rig, like the MI6 rig, developed and realized by Trenitalia. The parameters that mainly influence the algorithm precision are the wheel/rail adhesion, the type of train, the type of operation and the gradient of the line. For each parameter a series of classes has been defined and combined in order to obtain a set of tests able to represent the behaviour of the algorithm in all the cases it could meet in reality and then to obtain a reliable estimation of its prevision. In this work the criteria used to define the data set will be described. Then this set will be used to test the odometry algorithm developed for the SCMT, the Italian ATP system and the results will be presented.
\end{abstract}




\section{Introduction}

An Automatic Train Control system provides a high level of train safety, allowing one to increase the running speed and the line capacity. These systems automatically perform their monitoring and control action in order to prevent against the consequences of driver errors. Usually an ATP system includes three components:

- $\quad$ the wayside equipment, which generates codes to be transmitted to the train;

- the track-to-train transmission system, which transmits information from the wayside equipment to the train;

- the train on board equipment, which elaborates the information received from the track and from sensors mounted on the train and decides the actions to be performed.

The track subsystem gives to the train a series of information, for instance:

- the current position and the line gradient;

- the distance to targets (point in correspondence of which speed restrictions have to be achieved);

- the target speed (i.e. the speed that has not to be exceeded when the train passes through a target point).

This information is communicated to the train with the aid of fixed balises or another kind of absolute information. Between two consecutive information points on the line, the on-board subsystem perform a dead reckoning of the speed and the travelled distance and calculates:

- the minimum distance that allows to respect the speed restrictions at the next objective points: this value depends on the actual train speed, on the braking parameters and on the objective speed;

- the distance to the next information points.

If the difference between the distance to one or more of the next objective points and the distance that allows to respect the speed restriction is smaller than a fixed value, the on-board subsystem intervenes, for instance by activating the emergency braking.

A correct estimate of distance to target and actual velocity is then crucial to evaluate residual braking resources, in terms of available deceleration, in order to reach the targets at the required speeds.

In order to perform this estimate, some of the ERTMS implementations (European Railways Traffic Management System, the system under development for the European railways) uses a set of sensors including: encoders positioned on two independent wheels, a radar sensor positioned on the first vehicle case, and a longitudinal accelerometer. 
The performance of dead reckoning by means of odometry (in terms of precision in the estimation of speed and travelled distance) depends on how the information from different types of sensors is fused. For example, the estimation obtained with the measure of one or more wheel speeds may fail if degraded adhesion in the wheel-rail interaction occurs, due to rain, fog, ice, leaves, and so on, and the train is accelerating or braking, i.e. when pure rolling conditions between the wheel and the rail do not hold anymore, and macroscopic sliding occurs on one or more of the axles equipped with odometry sensors. The accelerometer sensor is sensitive to the inclination with respect to the horizontal direction, so it can give erroneous information in presence of a line gradient or pitch motion of the vehicle where it is mounted.

\section{The odometry system}

This module named odometry system evaluates the speed and train position during its run. It includes a series of sensors (for example wheel angular speed sensors, radar Doppler, longitudinal accelerometer) and a procedure (named "Odometry Algorithm"), that fuses data from the track and from the sensors mounted on the train in order to perform the odometric estimation. The values calculated by the odometric system are communicated to the ATP on board module, that compares them with the braking curves and decides an eventual intervention.

The odometry system performance, in terms of accuracy of speed and distance estimation, may affect the whole ATP system performance, in particular:

- if the odometry system tends to under-estimate the speed and the current position, the safety of the ATP system may be affected, since the train "thinks" to have a speed lower than the real one and/or to be at a distance from the objective greater than the real one, then a possible intervention of the control system could be delayed and the objective (for example stopping at a red signal) could be not respected;

- $\quad$ on the other hand, if the odometry system tends to over-estimate the speed and the current position, the efficiency of the ATP system may be affected, in this case the train "thinks" to have a speed greater than the real one and/or to be at a distance from the objective shorter than the real one, then a possible intervention of the control system or could be not necessary or anticipated.

For these reasons a particular care have to be put in the design and testing of such devices.

\section{Sensors used to perform the odometric estimation}

\subsection{Wheel speed sensors}

The sensors that measure the wheel angular speed are widely used in the on board subsystems (for example WSP and anti slip devices). Parameters limiting 
the accuracy of this kind of measure are the resolution of the sensor, the sampling time, electrical noise, mechanical imperfections such as backlash and, most importantly, slip and slide between the wheel and track.

Some research has been carried out to find methods for correcting the speed and position error caused by slip and slide using wheel angular speed sensors. The odometry algorithm developed for the SCMT (Sistema di Controllo Marcia Treno), the Italian ATP system, is based on the measures obtained from two wheel angular speed sensors. The algorithm is able to detect when the wheels are sliding and to then to properly correct the speed estimation [5-7].

\subsection{Accelerometer}

Linear accelerometers can be used to measure the linear acceleration of the vehicle, by integrating the acceleration signals both speed and position data can be derived. For this measurement method the exact initial values of speed and position are required.

There are many types of possible errors that could arise using this type of sensor, in particular, the measure is sensitive to the angular position of the sensor and then to the motion of the body on which it is mounted. For example, if a mono-axial accelerometer is used to measure the longitudinal acceleration of the train, the measure will be affected by the pitch motion of the vehicle and by the line gradient.

\subsection{Radar Doppler}

This type of sensor measures the relative speed between itself and a surface by detecting the frequency shift of a signal. This phenomenon is known as the Doppler effect. The measure of this kind $\mathrm{f}$ sensor may be affected by some possible sources of error:

- very smooth surfaces may cause loss of discrimination in the reflected signal,

- change in the radiation angle caused by the pitch and tilting motion of the body on which the sensor is mounted,

- existence of vibration can create noise in the data,

- existence of radar noise and bias errors.

\subsection{Sensor fusion}

In order to obtain a reliable odometric estimation and to prevent system failure the use of different types of sensors is necessary. The different measures has to be combined, the system has to identify the conditions in which the sensors are operating and to choose the measure that, in every situation, has the maximum reliability.

The odometry algorithm is then designed to perform an estimation of the state of the system (it has to be able to recognize the situations in which one or more of the sensor measures are affected by an error due to the operational conditions) 
and to choose for every condition, the estimation procedure that with the greater probability is the more corrected.

The following table summarizes the sensitivity of each sensor with respect to particular operational conditions.

Table 1: $\quad$ Sensor measure sensitivities.

\begin{tabular}{|c|c|c|c|}
\hline Type of sensor & Accelerometer & $\begin{array}{l}\text { Angular speed } \\
\text { sensors }\end{array}$ & Radar Doppler \\
\hline \multicolumn{4}{|l|}{ Sensitivity with respect to } \\
\hline $\begin{array}{l}\text { Adhesion between the wheels } \\
\text { and the rail }\end{array}$ & $\mathrm{NO}$ & YES & \multirow{6}{*}{$\begin{array}{l}\text { It is not possible to } \\
\text { find a relation } \\
\text { between the radar } \\
\text { Doppler sensor } \\
\text { performance and } \\
\text { these conditions }\end{array}$} \\
\hline $\begin{array}{l}\text { WSP and pneumatic brake } \\
\text { dynamics }\end{array}$ & $\mathrm{NO}$ & YES & \\
\hline WSP and electric brake & $\mathrm{NO}$ & YES & \\
\hline $\begin{array}{l}\text { Traction system, traction } \\
\text { control (anti slip device) }\end{array}$ & $\mathrm{NO}$ & YES & \\
\hline $\begin{array}{l}\text { Mechanical properties of the } \\
\text { vehicle and of the traction } \\
\text { system }\end{array}$ & $\mathrm{NO}$ & YES & \\
\hline Information on line gradient & YES & $\mathrm{NO}$ & \\
\hline Vehicle pitch motion & YES & NO & YES \\
\hline Vehicle roll - tilting & NO & NO & YES \\
\hline Reflecting properties of the line & NO & NO & YES \\
\hline $\begin{array}{l}\text { Presence of contaminants } \\
\text { (snow, water, dead leaves etc.) }\end{array}$ & $\mathrm{NO}$ & NO & YES \\
\hline
\end{tabular}

\section{Design of experiments}

The test of the odometry subsystems could be performed by a series of test runs, but this way is practically difficult for these reasons:

- a great number of tests are necessary in order to properly validate device performances;

- $\quad$ some testing conditions cannot be obtained during a run on the rail;

- testing conditions are difficultly controllable and repeatable;

- the testing activities have to be integrated with the normal rail traffic.

This way is not practically suitable in particular in the design phase of the system, when the estimation strategy has to be optimized. In this phase a great amount of data is necessary in order to explore all the operational scenarios that the system could meet in the reality.

Another testing strategy, that effectively can overcome all these problems, is represented by a proper HIL test rig. The feature of the test rig used to test the ATP on board subsystems is described in [1-3].

If only the odometry algorithm has to be validated, the test rig can be used in a off-line mode, in other words the generation of the test cases can be independent from the validation phase. Then, once the type and the number of tests have been decided, a single data base of tests has to be defined. The availability of this data set allows to speed up the testing phase, to have a useful 
tool able to compare the performance of different solutions, to perform easily sensitivity analysis and optimization procedures.

This way is obviously not suitable if the whole on board subsystem is tested, because the intervention of the ATP system modify the dynamics of the test.

The tests used to validate the odometry system have to represent all the operative conditions that the system could meet during its activity. In the tests the situations that could affect the safety of the system (situations in which one or more sensor measures are affected by errors and uncertainties) have to be reproduced, in order to test the safety o the system. In order to define a proper set of test cases, an analysis of parameters that mainly influence odometric estimation performance is necessary.

From the analysis of the sensor features the main parameters that affect system performances were identified:

- train type and composition: it has an effect on the wheel dynamics (in presence of poor adhesion conditions) and on the dynamics of the lead vehicle, this parameter then has an effect on wheel speed sensor, accelerometer and radar Doppler measures;

- brake mode: it has an effect on the dynamics of the brake propagation and then on wheel dynamics (in presence of poor adhesion conditions) affecting the wheel speed sensor measure;

- train length;

- line gradient: this parameter has an effect on accelerometer sensor measures (a component given by the projection of the gravity acceleration on the travelling direction is added to the measure of the accelerometer, this component acts as a disturb on the measure and if it is not properly corrected may leads to high estimation errors);

- adhesion conditions: they have an effect on the wheel dynamics and on the dynamics of the lead vehicle, this parameter then has an effect on wheel speed sensor, accelerometer and radar Doppler measures.

The coefficient used to specify the adhesion conditions is the static friction coefficient, imposed at the beginning of the test. During the test this coefficient can change, due to the wheels sliding and their time history.

For each parameter the main significant categories were identified. By the combination of all these cases the set of tests necessary to completely analyse the algorithm behaviour were defined [1]. Once the tests have been realized, the results can be analysed in order to better understand which parameters have an effective influence on system performance and how these parameters affect the precision of the estimation.

Once the test conditions have been defined, the methods for the result elaboration have to be set. Since the number of data is very high the odometric estimation error was evaluated by means of statistical techniques. For each simulation, the travelled distance is divided in elementary segments with a fixed length (for example 200, 500 and $1000 \mathrm{~m}$ ) and the difference between the estimated distance and the reference value is calculated. At the end of this task three sets of data are available, the results can be compared with the system specifications. 
Table 2: $\quad$ Parameters used to design the tests.

\begin{tabular}{|l|}
\hline Train type and composition \\
\hline Locomotive, empty freight train \\
\hline Locomotive, loaded freight train \\
\hline Locomotive, passenger train \\
\hline Coach, passenger train, traction applied to the end of the train \\
\hline Train length \\
\hline Minimum value (on the basis of braked weight percentage) \\
\hline Maximum value \\
\hline Intermediate value between the preceding ones \\
\hline Line gradient \\
\hline 0 \\
\hline $\pm 9 \%$ \\
\hline $\pm 21 \%$ \\
\hline $\pm 35 \%$ \\
\hline Adhesion between the wheel and the rail $\mu$ \\
\hline 0.09 \\
\hline 0.07 \\
\hline 0.05 \\
\hline 0.03 \\
\hline Type of operation \\
\hline $\begin{array}{l}\text { A: Acceleration from } 0 \mathrm{~km} / \mathrm{h} \text { up to } 100 \mathrm{~km} / \mathrm{h} \text { with the maximum traction torque available (that } \\
\text { depends on the adhesion value), three minutes at constant speed and emergency braking }\end{array}$ \\
\hline $\begin{array}{l}\text { B: Emergency braking from a speed included in the range between the maximum speed and } 20 \\
\text { km/h }\end{array}$ \\
\hline $\begin{array}{l}\text { C: } \text { Maximum service braking from a speed included in the range between the maximum speed } \\
\text { and } 20 \mathrm{~km} / \mathrm{h}\end{array}$ \\
\hline $\begin{array}{l}\text { D: Minimum service braking from a speed included in the range between the maximum speed and } \\
20 \mathrm{~km} / \mathrm{h}\end{array}$ \\
\hline $\begin{array}{l}\text { E: Acceleration from } 0 \text { up to the maximum speed with the } 25,50, \text { and } 75 \% \text { of the available traction } \\
\text { torque }\end{array}$ \\
\hline $\begin{array}{l}\text { F: Maximum/minimum service braking from the maximum allowed speed up to } 50 \% \text { of the } \\
\text { maximum speed, followed by an acceleration with the } 25,50,75,100 \% \text { of the available traction } \\
\text { torque }\end{array}$ \\
\hline $\begin{array}{l}\text { G: Acceleration with the } 25,50,75,100 \% \text { of the available traction torque followed by an } \\
\text { emergency braking }\end{array}$ \\
\hline
\end{tabular}

\section{Examples}

The following figures show an example of test. Figure 1 shows the actual and estimated speed and the velocities where the speed sensors are mounted. Figure 2 shows the difference between estimated and actual speed in the test. A single locomotive running on a line with degraded adhesion conditions (reference value 0.05 ) and no gradient was simulated in this test. The test was carried out using a odometry algorithm that uses two wheel angular speed sensors and a longitudinal accelerometer on the vehicle body. As it can be seen, even if the test conditions are extremely severe, the odometry algorithm error is quite small (the maximum value is about $1.5 \mathrm{~m} / \mathrm{s}$ ). Figure 2 shows that the error on speed estimation increases during a braking phase, with an approximately linear law. This increasing is due to the fact that the accelerometer measure is affected by the 
pitch motion of the body where the sensor is mounted (in this case the locomotive). However, during a braking phase the algorithm tends to over-estimate the speed, so the odometer error does not decrease the system safety.
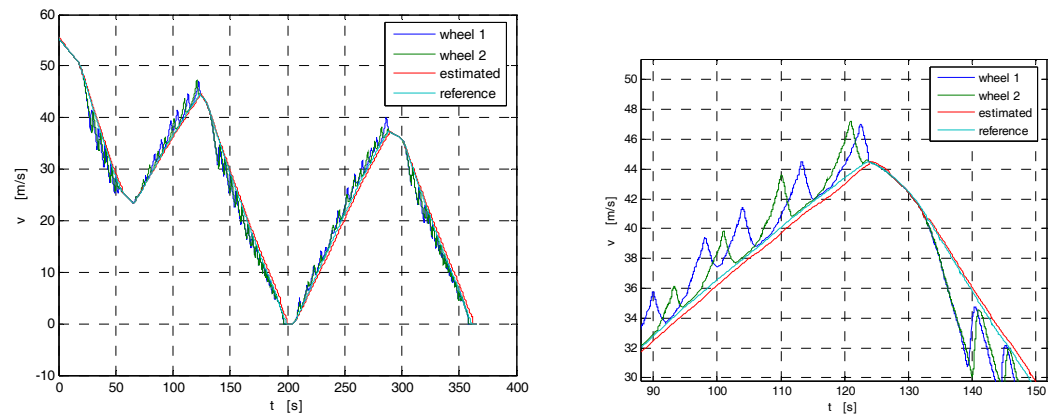

Figure 1: Actual and estimated speed.

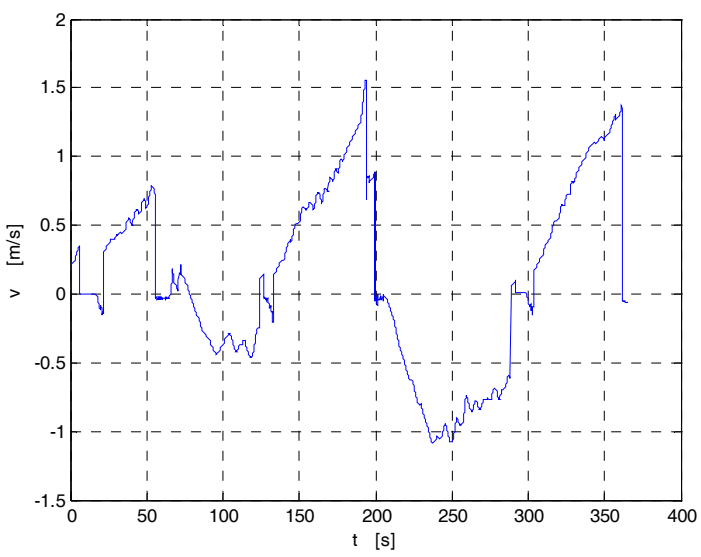

Figure 2: Difference between the estimated and the actual speed.

Figure 3 shows an example of the odometric error distribution, compared with a normal distribution. The example is relative to an algorithm that tends to over estimate the travelled distance, and then the distribution is quite different from the normal one.

\section{Conclusions}

In the ATP/ATC systems, a correct estimate of distance to target and actual velocity is fundamental to evaluate residual braking resources, in terms of available deceleration, in order to reach the targets at the required speeds. This device has to be properly designed and validated, in order to quantify its performance. 


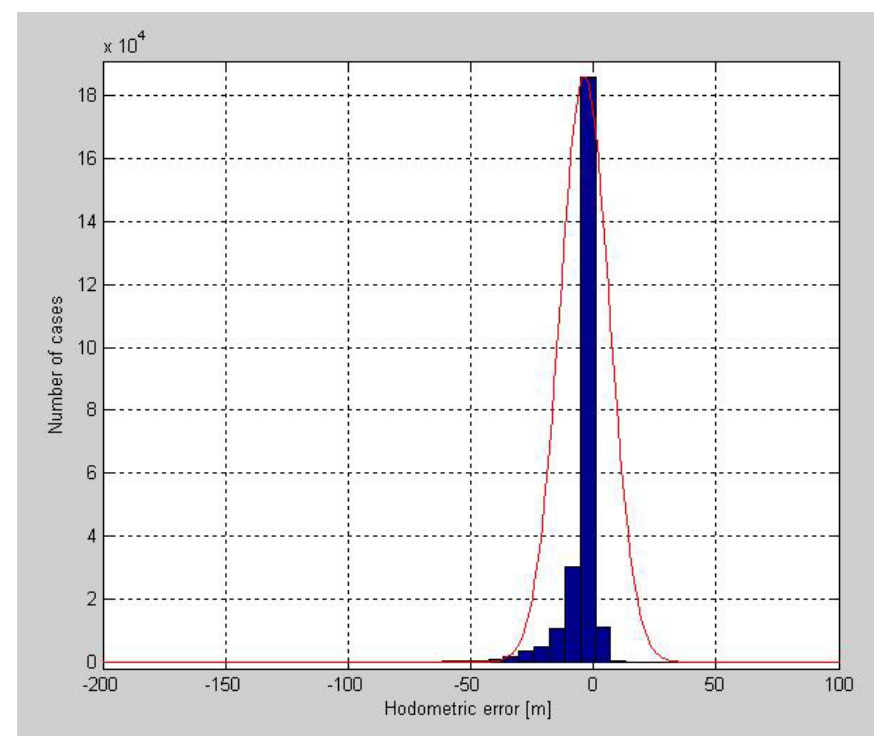

Figure 3: Statistical distribution of the odometric error.

The use of a properly designed HIL test rig can effectively represent an interesting solution for the testing activities necessary to the validation of odometry devices. This test methodology allows to greatly simplify the validation procedure. Test runs on the line are no more necessary and then the costs decreases sensibly. Testing conditions are completely controllable and repeatable this permits for example the comparison between different solutions. Extreme testing conditions (very low adhesion conditions, axles locking up and so on), that are difficult or dangerous to be reproduced on the line, can be easily obtained.

From the analysis of the type of sensors and the algorithm used to perform the odometric estimation the main parameters that may have an influence on system performance were identified. For each parameter a series of classes were defined. The set of data usable for the testing activity was then defined by combining the different classes.

\section{References}

[1] F. Addeo, B. Allotta, M. Malvezzi, L. Pugi, A. Tarasconi, M. Violani, "ATP/ATC subsystem testing and validation using a HIL test rig", Proc. of 9th Int. Conf. on Computer Aided Design, Manufacture and Operation in the Railway and Other Advanced Mass Transit Systems, May 2004, Dresden, Germany.

[2] P. Toni, B.Allotta, A.Rindi, Un approccio innovativo alla progettazione di banchi prova per sistemi ferroviari, Ingegneria ferroviaria, Aprile 2003, n. 4, pp.341-347. 
[3] B. Allotta, M. Malvezzi, L. Pugi, M. Rinchi, A. Rindi, P. Toni, A. Amore, R. Cheli, G. Cocci, P. Presciani, G. Puliatti, "A Test Rig for Evaluating Odometry Algorithms," Proc. of 8th Int. Conf. on Computer Aided Design, Manufacture and Operation in the Railway and Other Advanced Mass Transit Systems, 12 - 14 June 2002, Lemnos, GR.

[4] TRENITALIA S.p.A. Unità Tecnologie Materiale Rotabile "SCMT Progetto dell'algoritmo per il calcolo della velocità istantanea del treno e dello spazio percorso," UTMR/DT.S.PS, 31/10/2000.

[5] TRENITALIA S.p.A. Unità Tecnologie Materiale Rotabile "Odometria SCMT:Principi generali dell'algoritmo per il calcolo della velocità stimata in caso di pattinamento o slittamento degli assi di misura: Specifica Requisiti Funzionali.” Specifica N. 372574 esp. 03, 28/11/2002.

[6] TRENITALIA S.p.A. Unità Tecnologie Materiale Rotabile "Odometria SCMT:Principi generali dell'algoritmo per il calcolo della velocità stimata in caso di pattinamento o slittamento degli assi di misura: Specifica Requisiti Software” Bozza 4 Specifica N. 373450 esp. 00, 29/11/2002. 